\title{
Biomechanical structure of sprint start and effect of biological feedback methods on sprint start performance
}

\author{
Ilbilge OZSU
}

Institute of Health Sciences, Ege University, İzmir, Turkey, e-mail: ilbilgeozsu@gmail.com

\begin{abstract}
One of the profound factors that affect sprint performance of athletes is the transfer of the possible highest propulsive force onto the starting blocks using an ideal sprint start body position. Hence, in the literature, there are a great deal of studies related to assessment and improvement of sprint start performance. In this review, evaluation of the literature based on improving the sprint start performance and sharing obtained instructions with sport scientists, trainers and athletes were aimed. According to the literature knowledge, it is stated that higher propulsive force onto the starting block and acceleration are two most important factors affecting on the results of sprint running. Also, it is rational to state that permanent kinesthetic awareness of individualized sprint start position could lead to significant improvements in sprint performances. For this reason, biofeedback trainings will be useful methods that provide a kinesthetic awareness of individualized sprint start position for athletes. Thus, athletes increase the probability of learning motor skill when they have opportunity to compare the actual motor performance output with expected ideal performance output. It is stated that motor skill acquisition level of athletes increases considerably if feedback is provided appropriately. Accordingly, the aim of this review is to present literature based knowledge about biomechanics of sprint start and effects of biological feedback methods on sprint start performance.
\end{abstract}

Keywords: Biofeedback, biomechanics, sprint performance, starting block.

\section{INTRODUCTION}

Trainers consistently seek for new methods to improve the performance level of athletes (48). Improvement of athletic techniques, with which motor skills are performed, is directly related to learning ability of athlete (50). Therefore, trainers should create an efficient learning environment for their athletes during the training process. In recent years, use of information technology is integrated into sports field and outputs of this technology are used in the design of training programmes. This technology allows athletes to compare internal data (own body-related information) with external data (actual movement-related information) that facilitates learning and/or adjustment process of motor skills, thus leads to sports-related technical improvement. In this brief review, information technology used in the field of sports and rehabilitation are summarized. Biomechanical methods used in the analyses of sprint start performance, in which information technology is extensively used, and factors affecting sprint start performance constitute the main subject of this review.
Sports biomechanics is a branch of sports sciences that investigates the sports performance and mechanical characteristics of causes affecting on sports performance (22). One of the crucial aims of sports biomechanics is to provide scientific knowledge about how to optimize movement technique in a sports related motor skill. Various high-tech devices are used in biomechanical analyses and these devices provide performance outputs based on basic equations of Newtonian mechanics. These outputs could be used in the enhancement of sports performance $(18,34,36)$, identification of improper movement techniques (17), mastering proper movement techniques (49) and minimization of injury risk $(15,38,43)$.

\section{METHODOLOGY}

This review was conducted using the following electronic databases: Medline, PubMed, ISI Web of Knowledge and Scopus. Key search words included, sprint start, starting blocks, Biofeedback, Biomechanics, Sprint Performance, propulsive force, force plate, camera, neuromuscular activation, EMG,. Articles were checked for relevant content and were included based on the following criteria: 1) 
Published in English, 2) the participants were healthy, unhealthy and trained.

\section{Biofeedback method}

Biofeedback method is a rapidly developing method and its history goes back to late 1960s (4). Several electronic devices equipped with sensors and transducers are used in the biofeedback method with the aim of assessing and monitoring some biological responses that an individual cannot be aware of or cannot control voluntarily such as heart rate, muscle tone, skin temperature, brain activities etc. (51). Biofeedback data include different types of biofeedback signals such as numeric, auditory, visual or proprioceptive.

Proprioceptive biofeedback is a complex method that includes perpetual coordination between nervous system and musculo-skeletal system which is based on the awareness of motor skill being performed. Proprioceptive biofeedback is defined as the individual's perception of the movement and spatial orientation of body segments in relation to each other without the support of organs of vision, touch and balance (20). Proprioceptive sense includes the combination of muscle spindle, golgi tendon apparatus, joint angle sensors, skin mechanoreceptors and their afferent nerve pathways $(9,10)$. Successful sports performance could only be achieved in the case of straight and smooth performance of targeted motor skill. These mechanoreceptors and proprioceptors which have complex neuronal operating mechanisms, serve to prevent irregular movements during the execution of motor skill and ensure that movements of body parts follow the targeted trajectories. These trajectories show resistance to any kind of perturbation. Motor commands are adapted via visual and proprioceptive feedback in response to possible perturbations in order to ensure that motor skill is performed according to pre-planned trajectories. In addition, performing a motor skill within a pre-planned range of motion is dependent on the success of this feedback mechanism.

An adaptive, feed-forward control mechanism (motor adaptation) is responsible for the rapid adjustment of any kind of body part-related trajectory errors resulted from unexpected mechanical perturbations $(21,42)$. These body partrelated trajectory errors and perturbations experienced during the performance of any kind of motor task are kept in the memories of individuals and these information are used to perform the movements during the motor task more smoothly and straightly $(39,46)$.

\section{Biofeedback methods in the fields of sports science and rehabilitation}

It is stated that motor skill acquisition level of athletes increases considerably if feedback is provided appropriately (40). According to this opinion, it could be concluded that feedback methods are basic factors in the enhancement of motor skill performance. However, feedback methods could only be applied to individuals who are aware of the performance goal and willing to carry out specific corrections relative to predetermined performance outcomes (23).

Liebermann et al. (23) investigated various information technologies used for providing appropriate feedback to cyclists, downhill skiers, shooters, goalkeepers and gymnasts. They compared visual, auditory and proprioceptive feedback systems with each other. Feedback is reported to be the basic factor in the enhancement of athletic performance considering feedback-related studies available in the literature. Liebermann et al. (23) stated that recent developments in information technology make it possible to provide athletes with feedback during training and competition. Thus, athletes find opportunity to enhance their performance level and correct their errors related to performed motor skills. Basic external feedback and auxiliary technologies (from simple camera recordings to complex simulations) are reported to have profound effects on motor learning that Therefore, it is suggested that this issue should be considered with great emphasis in the design of training plans (23).

Fothergill (12) showed positive effects of feedback on rowing performance of 5 amateur rowers within the age range of 25-41. Fothergill (12) used real-time visual feedback method during rowing performance on a rowing ergometer. This feedback device detected actual rowing movements of the rowers and provided simultaneous visual feedback -together with kinetic data- representing the ideal rowing technique identified by the trainer. The feedback device included two 3-D cameras that detected rowing movements, an interface transmitting the visual data to a computer, a monitor placed in a position that was easily seen by the rowers and some auxiliary devices. At the end of the study, a questionary was applied to rowers. According to this questionary, it was concluded that the use of this real-time visual feedback method did 
not interrupt the performance of rowers and rowers perceived that they performed with a better technique when they received real-time visual feedback (12).

There are two crucial stages that determine the success of performance during the ballistic movements in archery and shooting. Athletes keep their respiration rhythm and heart rate under control, they stabilize their body, minimize the body sway and maintain a balanced posture, which constitutes the first stage. The quality of this balanced posture can be identified by tracing the pressure changes related to center of pressure of the athlete while he/she is performing the related motor task on a force plate; the greater the distribution area of the projection graphic of the athlete's center of pressure, the lower the balance quality of the athlete. The second stage requires the athlete to focus visually on the arrow or gun. In this stage, a laser beam can be used as a source of feedback. This feedback method ensures that athlete focuses on the center of a specified target via a computerized system. In the cases that focus of the athlete deviates from the center of the target, system provides an auditory signal. This signal consists of a sound whose frequency increases in direct proportion to the magnitude of the deviation. In this method, athlete tries to perform the motor skill comparing the internal feedback signals (proprioceptive feedback) with external feedback signals (auditory feedback) (23).

Barrios et al. (2) conducted a study on 8 individuals (age range 18-35) with varus deformity. In their study, they used real-time feedback method to co-ordinate the distorted knee joints). They aimed to reduce the moment of knee adduction to coordinate the distortion. This reduction and systematic training programs designed to adjust the structure of the knee joint were assumed to provide more natural and smoother knee movement pattern. An 8-week real-time feedback training period was conducted to test the above mentioned assumptions. Individuals received visual feedback during the movement of knee joint in the frontal plane and received verbal feedback during the internal rotation and adduction of hip joint to reduce the adduction in the knee joint. Investigators compared pretraining and post-training walking patterns. They identified the adduction and internal rotation of hip joint, and adduction of knee joint and knee joint adduction moment. After the completion of the training period, increases of $8^{\circ}$ and $3^{\circ}$ were observed in internal rotation and adduction of hip joint, respectively, in individuals whose walking pattern was changed. Moreover, knee adduction and knee adduction moment were decreased by $2^{\circ}$ and $19 \%$, respectively. No change was observed in the walking pattern of three individuals. Individuals having changes in their walking pattern reported that they felt more comfortable and perceived less effort during walking after the feedback training period (2).

Robotic devices produced for walking-related rehabilitation programs provide patients with detailed instructions during their walking training. Feedback provided during robotic-supported walking training, which is based on motor learning principles, helps patients to regain their walking ability. Banz et al. (1) investigated the effects of visual feedback training on rehabilitation process of 12 patients who had neurologic walking disorder arising from spinal cord injury. Investigators reported that visual feedback received from a computer made a significant contribution to roboticsupported walking training (1).

Above mentioned studies put forth the positive effects of feedback in the fields of sports sciences and rehabilitation. Similar innovatory and technological methods were also used with the aim of enhancing athletic performance in sprint runs. This review, in particular, focused on investigating effects of various feedback methods including hightech devices on sprint start performance.

\section{Factors Affecting Sprint Start Performance}

The history of use of starting block in sprint runs goes back to late 1920s (47). Various studies using kinematic, kinetic and muscular activation data have been conducted since those years to investigate the sprint start techniques of sprint athletes with different performance levels.

Sprinting success in $60 \mathrm{~m}, 100 \mathrm{~m}, 200 \mathrm{~m}$ and 400 $\mathrm{m}$ sprint runs is directly related to sprint start and block acceleration; two crucial phases of a sprint run. Hence, many investigators studied the biomechanical components of these two phases to clarify the determinants of sprint velocity $(13,16,29,30,33,41,45)$.

Sprint start and transition to acceleration phase constitute a complex movement series. This movement series requires high-level muscle activation and effective coordination of cyclic and acyclic movements (6). Sprint athletes try to obtain a maximal block velocity during the phases of sprint start and block acceleration. Block acceleration is the 
first phase of a sprint run during which kinetic parameters of the first step of the sprint change dynamically. Block acceleration is a complex cycling movement during which step length, step frequency, position of the center of mass at the foot contact, duration of foot contact and duration of flight phase change rapidly. The contact phase has two componenets: braking and propulsion phases (25). The characteristics of contact and flight phases change as the biomechanical position of the sprint athlete changes. The lesser the duration of the contact phase, the greater the flight phase of the sprint athlete. Step length is dependent on the body height/leg length ratio and the total propulsive force applied by the extensor muscles of the hip (m. gluteus maximus), knee (m. vastus lateralis, $\mathrm{m}$. rectus femoris) and ankle joints (m. gastrocnemius) onto the starting blocks. Contact phase is the profound source of the velocity efficiency during the sprint run (30). Contact phase should be completed within the shortest time that is related to the optimal ratio between braking and propulsion phases. Step frequency depends on the genetic make-up the sprint athlete and the function of the central nervous system that allows greater number of muscle fibers to be recruited (31). As the step frequency increases, step length gets shorter and vice versa is true. An optimal ratio between the step length and step frequency of a sprint athlete determines the efficiency of block acceleration phase (8).

\section{Studies investigating sprint start performance}

McClements et al. (27) developed the Saskatchewan Sprint Start Device to provide sprint athletes with feedback and to enhance their sprint start performance. External feedback provided by the device had an easily understandable characteristic. They conducted the study on 62 athletes and investigated the effects of their feedback device on the sprint start performance (27). Athletes, training on a standard running field, received feedback from this device on the generated force and sprint times. McClements et al. (27) suggested different feedback models using this device. They concluded that one of the feedback models that depended on the maximal horizontal and vertical forces applied onto the starting blocks was the best model that determined the sprint performance (27). This model considered the maximal horizontal and vertical force data and the reaction time data of the rear foot.

Mendoza and Schollhorn (28) conducted a similar study on 8 young athletes. They aimed to improve sprint start technique of these athletes using a biomechanical feedback training method. They investigated three technical variables: 1) distance between starting block and the starting line, 2) knee joint angle of the front leg, 3) distribution of body weight on each hand (28). Horizontal block velocity and $10 \mathrm{~m}$ sprint time constituted the performance criteria of athletes. All variables were measured simultaneously and feedback was provided immediately after the evaluation of the performance measures (28). At the end of the study, 7 of 8 athletes changed their sprint start positions and statistically significant improvements were observed in their sprint start performances. In addition, a strong positive relationship was found between the generated forces at the onset of the sprint start and $10 \mathrm{~m}$ sprint times. Mendoza and Schollhorn (28) concluded that inter-individual differences existed in block velocity among athletes and suggested that biomechanical feedback should be used effectively during sprint start training .

According to findings obtained in the study of Mendoza and Schollhorn (28), it could be argued that motor task-related feedback training led to an increase in the kinesthetic awareness of athletes and accordingly, allowed them to adjust their sprint start position to achieve higher performance levels. Moreover, this biofeedback training could be regarded as an effective means of acquiring an efficient and permanent sprint start position.

Sprint athletes have different choices in the context of sprint start positions. These choices show high inter-individual variability. Schot and Knutzen (41) detected 4 different sprint start positions that were related to anthropometric characteristics of athletes. Biomechanical analyses of these positions were performed in their study using 12 athletes competing in hurdles sprint runs. They reported that wider placement of foots in the sprint start position, defined as "elongated starting position", led to higher propulsive force onto the starting block, increment in the first step distance and avarege velocity within 2 meters of the sprint (41). They could not set out in full the effects of hand placement on the sprint start performance. Findings of this study show that sprint start position varies depending on the anthropometric characteristics of athletes (41).

Coh et al. (5) conducted a study on 13 male (mean age of 24 years) and 11 female (mean age of 23 years) athletes and investigated the relationship between sprint start position and sprint start 
acceleration phase using technological methods. Kinetic variables of the onset of the sprint start and variables of acceleration phase were recorded by a two dimensional (2-D) kinematic video analysis system; kinetic variables of the onset of the sprint start were recorded by force plates placed on the start blocks. Time-related variables of sprint velocity and acceleration were measured by 4 photocells placed 5-10-20-30 $\mathrm{m}$ away from the starting line. Statistical analyses of sprint start velocities, reaction times and propulsive forces applied onto the starting blocks were performed and a significant difference was detected in kinetic variables of sprint start performance between male and female athletes. According to Čoh, et al. (5), differences in kinetic variables are more apparent than differences in kinematic variables between male and female athletes. In addition, start acceleration was reported to be not affected from kinematic and kinetic factors.

Coh et al. (5) suggested athletes to adjust their sprint start position according to reference points identified in the study of Schot and Knutzen (41). According to Schot and Knutzen (41) vertical distance between running track and center of body mass should be $54.4 \pm 6.2 \mathrm{~cm}$ in males and $53.2 \pm 2.0$ $\mathrm{cm}$ in females; horizontal distance between projection of center of mass and starting line should be $18.8 \pm 5.1 \mathrm{~cm}$ in males and $15.7 \pm 3.0 \mathrm{~cm}$ in females; knee joint angle of rear leg should be $112^{\circ} \pm$ $13.3^{\circ}$ in males and $115^{\circ} \pm 13.8^{\circ}$ in females. It was found that sprint start position and kinetic variables of the onset of the sprint start had effects on acceleration measures during the sprint. Schot and Knutzen (41) concluded that propulsive force applied onto the front starting block, reaction time and block velocity were the most crucial variables that should be developed to acquire an effective and efficient sprint start. It was also shown that significant differences were existed in set positions relative hip height and knee joint angle between males and females. In conclusion, Coh et al. (5) emphasized that sprint start position was unique for each athlete and showed variations based on motor skills of athletes and their morphological characteristics.

In another study of Coh et al. (8), a male athlete run five $20 \mathrm{~m}$ sprints and kinetic and kinematic variables that have direct effects on the results of sprint runs -motor reaction time, propulsive forces and starting block acceleration- were analyzed during these runs. High-speed 2-D camera, infrared photocell system and Opto Track Technology were used in the analysis of sprint start phase. It was found that higher the propulsive force applied onto the front starting block, the lower the motor reaction time (8). This led to improvement in the first step performance, thus increased block acceleration.

Kinetic energy of different body parts and angular velocity of different joints were investigated in the study of Slawinski et al. (44) conducted on 8 elite sprint athletes. Sixty three passive markers were placed on the reference points of athletes and each athlete performed 4 maximal $10 \mathrm{~m}$ sprints. These sprints were analyzed using an optoelectronic analysis system consisted of 12 digital cameras operating at 250 Hertz. It was detected that maximal speeds of athletes were dependent on flexion-extension, abduction-adduction and internalexternal rotation movements of the shoulders, chest and hip, not solely on the flexion-extension movement. Moreover, it was found that total maximal kinetic energy of the body was attained just before feet left the starting blocks. A higher level of synchronization between lower and upper extremities of athlets was suggested in order to improve the propulsive force applied onto starting blocks (44).

Bezodis et al. (3) conducted a study on 12 sprint athletes and stated that selection of criteria that are to be used in the assessment of sprint start performance is of great importance. They reported that maximal propulsive force applied onto the starting blocks is not only important for sprint start performance but also for each phase of sprint runs. Similarly, Mero et al. (33) stated that high maximal and average propulsive forces were key components of the high performances both in sprint start and early acceleration phase $(0-10 \mathrm{~m})$. Bezodis et al. (3) stated that normalized maximal propulsive force was an appropriate criterion that could be used in performance assessment of any phase of sprint runs. Findings of their study justified the importance of identifying the ideal sprint start position that allows athletes to apply maximal propulsive force onto the starting blocks (3).

Another study in the literature that investigated the sprint start was conducted by Harland and Steele (16) . Authors suggested a sprint start position with $90^{\circ}$ and $130^{\circ}$ knee joint angle of front leg and rear leg, respectively. These joint angles resulted in a relatively higher hip position compared to traditional sprint start positions. In addition, horizontal propulsive force applied onto the starting block was stated to have crucial importance to leave 
the starting blocks with maximal horizontal velocity and acceleration (16).

In his master thesis, Morrish (35) investigated the effects of progressive resistance training on acceleration obtained between the moments of sprint start and maximal velocity during a sprint run. He stated that the relationship between progressive resistance training and sprint start performance had a very complex structure (35).

Effects of muscle-tendon length on joint movements and joint forces during sprint start position were investigated in the study of Mero et al. (32). Nine male sprint athletes used starting blocks with $40^{\circ}$ and $65^{\circ}$ angle during their sprint runs. Horizontal velocity of the center of mass of athletes were analyzed and concluded that use of starting blocks with $40^{\circ}$ angle was resulted in better sprint start performances compared to use of starting blocks with $65^{\circ}$ angle. It was stated that $40^{\circ}$ angle led to greater muscle-tendon lengths of gastrocnemius and soleus muscles at the onset of the sprint start that resulted in higher ankle joint forces and moments (32). According to this study, these factors played an important role in obtaining a greater block velocity.

Guissard et al. (13) investigated the effects of front starting block slope on block velocity . Fourteen male and 3 female trained athletes participated in their study. Activities of medial gastrocnemius, soleus and vastus lateralis muscles were analyzed using electromyography (EMG) method during the sprint start with $30^{\circ}, 50^{\circ}$ and $70^{\circ}$ starting block angles. Starting block angles of $30^{\circ}$ and $50^{\circ}$ led to acute increases in lengths of medial gastrocnemius and soleus muscles. It was found that decrease in starting block angles resulted in increased block velocity. Guissard et al. (13) stated that athletes could enhance their block velocity and propulsive force by means of adjusting their extremity positions to attain an ideal sprint start position. It is important for sprinters to identify the optimal horizontal distances between front starting block and starting line as well as between each starting block. These optimal distances are crucial for maintaining the propulsion phase and for increasing contribution level of rear leg to propulsive force application onto the starting block. Guissard et al. (13) concluded that effects of starting block slopes -particularly front block- and joint angles on sprint start performance are well known, in contrary there is a great deficiency related to correlations between these variables and EMG responses of muscles during sprint start.

Coh et al. (6) conducted a study on a female sprinter with 13.19-second personal best in $110 \mathrm{~m}$ hurdles. They aimed to improve the sprint performance of this athlete depending on the results of performed biomechanical analysis. EMG data of 7 active muscles, basic kinematic and kinetic variables such as block velocity, acceleration during the first two steps and propulsive forces applied onto the starting blocks were analyzed. Gastrocnemius medialis muscle was shown to be the one of the most active muscles that had a great contribution to block acceleration and generation of propulsive force (6).

\section{DISCUSSION}

Results of biomechanical studies showed that sprint start positions show great inter-individual variation between sprint athletes $(5,6)$. It is seen that different sprint start positions were suggested in each different study $(5,16,27,28,41)$. These studies were conducted on highly different study samples (male, female, young, adult, amateur, elite athletes), accordingly results of the studies showed variations depending on the biomechanical, morphological and anthropometric characteristics of these athletes. It could be concluded that use of individualized training methods with individualized sprint start positions might provide athletes with important opportunities related to improvement of sprint performance. EMG analyses, particularly performed during the propulsion phase of the sprint start, should be also taken into consideration in the identification of precise sprint start position. Adapted versions of previously mentioned biofeedback methods that might be used during sprint start training sessions could constitute a profound factor in making the kinesthetic awareness of athletes related to ideal sprint start position permanent. It is rational to state that permanent kinesthetic awareness of individualized sprint start position could lead to significant improvements in sprint performances.

According to results of biomechanical studies conducted until now, reaction time, sprint start position and block velocity are identified as major factors contributing to sprint performance $(11,16,19,27,32,33)$. All these factors are integrated and each of them depends on central movement regulation process, biomotor abilities, energy production processes and morphological characteristics $(24,26)$. 
Reaction times of athletes raced in $100 \mathrm{~m}$ final in 2008 Beijing Olimpics were analyzed using highspeed cameras and results of these analyses showed that Usain Bolt, who broke a world record in this race, had a poor reaction performance (37). This issue raises a question about whether a good reaction time, which has been identified as a determining factor in the literature, is indeed important in sprint runs.

There are studies indicating that sprint athletes with higher performance levels are able to generate higher propulsive forces onto the starting blocks $(8,16,31,44)$. Mero et al. (32) showed that maximal rate of force development (highest force generated within the smallest time frame) was crucial in attaining maximal block velocity. Similarly, it was stated that propulsive force applied onto the starting blocks had direct effects on the block velocity and the first phase of acceleration after the sprint start $(14,32)$. According to literature knowledge, it could be concluded that sprint performance is dependent on the sprint start performance that is directly related to propulsive force applied onto the starting blocks $(16,19,27,30)$.

High correlation levels between start and block acceleration should be taken into consideration in the design of sprint start training programs (8). The starting position of sprint athlete that ensures the maximal block velocity is a prerequisite for faster sprint runs. Block acceleration is dependent on the length of the first step and the position of feet in the braking phase. The efficiency of block acceleration affects the biomechanical variables in the first ten steps of the sprint run.

In conclusion, according to the literature knowledge, it is stated that sprint start velocity and acceleration are two most important factors affecting on the results of sprint runs (5). Maximal and mean propulsive force applied onto the starting blocks are main components of sprint start performance as they are directly related to sprint start velocity and acceleration. Moreover, maximal and mean horizontal propulsive force generation capacities are said to be the main factor that seperates talented athletes from others (7). Therefore, ideal sprint start position that leads to maximal and mean propulsive forces should be assessed precisely considering inter-individual differences since this position is greatly affected by anthropometric measures of athletes $(8,41)$.

It is rational to conclude that extensive research should be performed on the improvement of information technology-related training methods to attain ideal athletic performances. Use of different feedback methods that facilitates learning of specific motor skills could enhance athletic performance in different sport branches as well as in sprint runs.

\section{KAYNAKLAR}

1. Banz R, Bolliger M, Colombo G, Dietz V, Lunenburger L. Computerized visual feedback: An adjunct to robotic-assisted gait training. Phys Ther, 2008; 88(10): 1135-1145.

2. Barrios JA, Crossley KM, Davis IS. Gait retraining to reduce the knee adduction moment through real-time visual feedback of dynamic knee alignment. J Biomech, 2010; 43(11): 2208-2213.

3. Bezodis NE, Salo AI, Trewartha G. Choice of sprint start performance measure affects the performance-based ranking within a group of sprinters: Which is the most appropriate measure? Sports Biomech, 2010; 9(4): 258-269.

4. Blumenstein B, Eli MB, Tenenbaum G. Brain and body in sport and exercise biofeed back applications in performance enhancement. 1st ed. Sussex: John Wiley \& Sons., 2002:1

5. Coh M, Jost B, Skof B, Tomazin K, Dolenec A. Kinematic and kinetic parameters of the sprint start and start acceleration model of top sprinters. Gymnica, 1998; 28: 33-42.

6. Coh M, Peharec S, Bacic P. The sprint start: Biomechanical analysis of kinematic, dynamic and electromyographic parameters. New Studies in Athletics, 2007; 22(3): 29-38.

7. Coh M, Peharec S, Bacic P, Kampmiller T. Dynamic factors and electromyographic activity in a sprint start. Biology of Sport, 2009; 26(2): 137-147.

8. Coh M, Tomazin K, Štuhec S. The biomechanical model of the sprint start and block acceleration. Facta Universitatis Physical Education and Sport, 2006; 4(2): 103-114.

9. Collins DF, Prochazka A. Movement illusions evoked by ensemble cutaneous input from the dorsum of the human hand. J Physiol, 1996; 496 (Pt 3)(857-871.

10. Collins DF, Refshauge KM, Todd G, Gandevia SC. Cutaneous receptors contribute to kinesthesia at the index finger, elbow, and knee. J Neurophysiol, 2005; 94(3): 1699-1706.

11. Coppenolle VH, Delecluse C, Goris M, Seagrave L, Kraayenhof $H$. An evaluation of the start action world class female sprinters. Track Technique, 1989; 90: 3581-3582.

12. Fothergill S. Examining the effect of real-time visual feedback on the quality of rowing technique. in 8th Conference of the International Sports Engineering Association (ISEA) 2010. Cambridge, UK: Procedia Engineering.

13. Guissard N, Duchateau J, Hainaut K. Emg and mechanical changes during sprint starts at different front block obliquities. Med Sci Sports Exerc, 1992; 24(11): 1257-1263.

14. Gutierrez-Davilla M, Dapena J, Campos J. The effect of muscular pre-tensing on the sprint start. J Appl Biomech, 2006; 22(3): 194-201.

15. Hamill J, van Emmerik RE, Heiderscheit BC, Li L. A dynamical systems approach to lower extremity running injuries. Clin Biomech (Bristol, Avon), 1999; 14(5): 297-308.

16. Harland MJ, Steele JR. Biomechanics of the sprint start. Sports Med, 1997; 23(1): 11-20. 
17. Hiley MJ, Yeadon MR. The margin for error when releasing the high bar for dismounts. J Biomech, 2003; 36(3): 313-319.

18. Hiley MJ, Yeadon MR. Optimisation of high bar circling technique for consistent performance of a triple piked somersault dismount. J Biomech, 2008; 41(8): 1730-1735.

19. Korchemny R. A new concept for sprint start and acceleration training. New Studies in Athletics, 1992; 7(4): 65-72.

20. Kuchenbecker KJ, Gurari N, Okamura AM. Effects of visual and proprioceptive motion feedback on human control of targeted movement. in In Proceedings of the International Conference on Rehabilitation Robotics. June, 2007. Noordwijk.

21. Lackner JR, Dizio P. Rapid adaptation to coriolis force perturbations of arm trajectory. J Neurophysiol, 1994; 72(1): 299-313.

22. Lees A. Science and the major racket sports: A review. J Sports Sci, 2003; 21(9): 707-732.

23. Liebermann DG, Katz L, Hughes MD, Bartlett RM, McClements J, Franks IM. Advances in the application of information technology to sport performance. J Sports Sci, 2002; 20(10): 755-769.

24. Locatelli E, Arsac L. The mechanics and energetics of the 100m sprint. New Studies in Athletics, 1995; 10(1): 81-87.

25. Luhtanen P, Komi PV. Force-, power-, and elasticity-velocity relationships in walking, running, and jumping. Eur J Appl Physiol Occup Physiol, 1980; 44(3): 279-289.

26. Mann R, Sprague P. A kinetic analysis of the ground leg during sprint running. Res Q Exerc Sport, 1980; 51(2): 334-348.

27. McClements, JD, Sanders, LK, Gande, BE. Kinetic and kinematic factors related to sprint starting as measured by the saskatchewan sprint start team. . New Studies in Athletics. 1996; 11(2-3): 133-135.

28. Mendoza L, Schollhorn W. Training of the sprint start technique with biomechanical feedback. J Sports Sci, 1993; 11(1): 25-29.

29. Mero A. Force-time characteristics and running velocity of male sprinters during the acceleration phase of sprinting. Research Quarterly for Exercise and Sport, 1988; 59(2):

30. Mero A, Komi PV. Reaction time and electromyographic activity during a sprint start. Eur J Appl Physiol Occup Physiol, 1990; 61(1-2): 73-80.

31. Mero A, Komi PV, Gregor RJ. Biomechanics of sprint running. A review. Sports Med, 1992; 13(6): 376-392.

32. Mero A, Kuitunen S, Harland M, Kyrolainen H, Komi PV. Effects of muscle-tendon length on joint moment and power during sprint starts. J Sports Sci, 2006; 24(2): 165-173.

33. Mero A, Luhtanen P, Komi P. A biomechanical study of the sprint start. Scandinavian Journal of Sport Sciences, 1983; 5(1): $20-28$.

34. Mills C, Yeadon MR, Pain MT. Modifying landing mat material properties may decrease peak contact forces but increase forefoot forces in gymnastics landings. Sports Biomech, 2010; 9(3): 153-164.

35. Morrish WA. Sprint start training, progressive resistance training and the ability to accelerate to maximum velocity. University of British Columbia, 1972.
36. Pau M, Loi A, Pezzotta MC. Does sensorimotor training improve the static balance of young volleyball players? Sports Biomech, 2012; 11(1): 97-107.

37. Graubner R, Nixdorf E. Biomechanical analysis of the sprint and hurdles events at the 2009 iaaf world championships in athletics. New Studies in Athletics, 2011; 26(1-2): 19-53.

38. Schache AG, Wrigley TV, Baker R, Pandy MG. Biomechanical response to hamstring muscle strain injury. Gait Posture, 2009; 29(2): 332-338.

39. Scheidt RA, Dingwell JB, Mussa-Ivaldi FA. Learning to move amid uncertainty. J Neurophysiol, 2001; 86(2): 971-985.

40. Schmidt RA, Lee T. Motor control and learning. ed. Champaign, IL: Human Kinetics, 1999:126-128

41. Schot PK, Knutzen KM. A biomechanical analysis of four sprint start positions. Res Q Exerc Sport, 1992; 63(2): 137-147.

42. Shadmehr R, Mussa-Ivaldi FA. Adaptive representation of dynamics during learning of a motor task. J Neurosci, 1994; 14(5 Pt 2): 3208-3224.

43. Silder A, Thelen DG, Heiderscheit BC. Effects of prior hamstring strain injury on strength, flexibility, and running mechanics. Clin Biomech (Bristol, Avon), 2010; 25(7): 681-686.

44. Slawinski J, Bonnefoy A, Leveque JM, Ontanon G, Riquet A, Dumas R, Cheze L. Kinematic and kinetic comparisons of elite and well-trained sprinters during sprint start. J Strength Cond Res, 2010; 24(4): 896-905.

45. Tellez T, Doolittle D. Sprinting from start to finish. Track Technique, 1984; 88: 2802-2805.

46. Thoroughman KA, Shadmehr R. Learning of action through adaptive combination of motor primitives. Nature, 2000; 407(6805): 742-747.

47. Ey W. Charles frederick "charlie" booth- inventor of starting blocks. The Veteran Athlete, 1987; 2(1): 4.

48. Waldron M, Worsfold P, Twist C, Lamb K. Concurrent validity and test-retest reliability of a global positioning system (gps) and timing gates to assess sprint performance variables. J Sports Sci, 2011; 29(15): 1613-1619.

49. Williams G, Irwin G, Kerwin DG, Newell KM. Kinematic changes during learning the longswing on high bar. Sports Biomech, 2012; 11(1): 20-33.

50. Yarrow K, Brown P, Krakauer JW. Inside the brain of an elite athlete: The neural processes that support high achievement in sports. Nat Rev Neurosci, 2009; 10(8): 585-596.

51. Zaichkowsky LD, Fuchs CZ. Biofeedback applications in exercise and athletic performance. Exerc Sport Sci Rev, 1988; 16: 381-421. 